\title{
Erratum to: Immunostimulatory activity of ConBr: a focus on splenocyte proliferation and proliferative cytokine secretion
}

Flávio de Oliveira Silva • Priscila das Neves Santos •

Cristiane Moutinho Lagos de Melo •

Edson Holanda Teixeira • Benildo Sousa Cavada •

Francisco Vassiliepe Sousa Arruda •

João Batista Cajazeiras • Alysson Chaves Almeida •

Valéria Rêgo Alves Pereira • Ana Lúcia Figueiredo Porto

Published online: 9 December 2011

(C) Springer-Verlag 2011

Erratum to: Cell Tissue Res (2011) 346:237-244

DOI 10.1007/s00441-011-1239-x

The original version of the article unfortunately contained a mistake. The order of authors on the published version was incorrect. The correct order of authors is shown above.

The online version of the original article can be found at http://dx.doi. org/10.1007/s00441-011-1239-x.

F. de Oliveira Silva A. L. F. Porto

Departamento de Morfologia e Fisiologia Animal,

Universidade Federal Rural de Pernambuco,

Recife, Pernambuco, Brazil

P. das Neves Santos

Centro de Ciências biológicas,

Universidade Federal de Pernambuco,

Recife, Pernambuco, Brazil

C. M. L. de Melo • V. R. A. Pereira

Departamento de Imunologia do Centro de Pesquisas Aggeu,

Magalhães - CPqAM/FIOCRUZ-UFPE,

Recife, Pernambuco, Brazil
E. H. Teixeira $\cdot$ B. S. Cavada

Departamento de Bioquímica e Biologia Molecular,

Universidade Federal do Ceará,

Fortaleza, Brazil

F. de Oliveira Silva $(\bowtie)$

Setor de Biotecnologia,

Laboratório de Imunopatologia Keizo Asami - LIKA/UFPE,

Av. Moraes Rego s/n, Cidade, Universitária,

50670-420 Recife, PE, Brazil

e-mail: foliveirasilva@gmail.com

F. V. S. Arruda $\cdot$ J. B. Cajazeiras · A. C. Almeida

Biomol-Lab, Departamento de Bioquímica e Biologia Molecular, Universidade Federal do Ceará,

Fortaleza, Brazil 